\title{
Sistema de Gestão Ambiental e Observação das Condições de Trabalho em uma Suinocultura em Campo Verde, Mato Grosso
}

\section{Analyze of an Environment System Management for Swine and Observation of working conditions}

\author{
${ }^{1}$ José Brito, ${ }^{2}$ Leonardo Zaque, ${ }^{3}$ Bruno Castro, ${ }^{4}$ Kerollayne Figueiredo, ${ }^{5}$ Stephany Guerra, ${ }^{6}$ Juliana Silveira. \\ ${ }^{123456}$ Pós-Graduandos em Engenharia de Segurança do Trabalho-Departamento de Engenharia Sanitária e \\ Ambiental Faculdade de Arquitetura, Engenharia e Tecnologia da Universidade Federal de Mato Grosso
}

Recebido em: fevereiro de 2016 aceito em: abril de 2016 publicado em: junho de 2016

Resumo:Resíduos são todas as substâncias consideradas indesejadas geradas na produção ou consumo de bens. Por meio da elaboração de um Sistema de Gestão Ambiental (SGA), pode ser gerenciado um sistema produtivo de forma a destinar corretamente os resíduos produzidos, além disso, há resíduos que podem ser reaproveitados para novas atividades.O correto gerenciamento deve considerar as questões ambientais e sociais de forma integrada, desse modo, as condições de segurança de trabalho são fator essencial para a sua correta operação. Os resíduos sólidos produzidos na Granja Bordignon, localizada em Campo Verde-MT, são separados e encaminhados a destinação adequada. Os resíduos domésticos são armazenados e encaminhados a coleta municipal, os recicláveis são coletados pela Cooperativa de catadores do município, os Resíduos de Serviços de Saúde são acondicionados e destinados a empresas especializadas em destinação final, os suínos mortos e material proveniente de origem orgânica são encaminhados a compostagem, os dejetos dos suínos são encaminhados para o sistema de tratamento de efluentes, composto por um Biodigestor seguido por duas lagoas. O gás gerado no biodigestor é destinado para um queimador tipo Flaar, e o efluente final do sistema resultante desse processo é utilizado como fertilizante orgânico. Este artigo tem por objetivo apresentar um SGA realizado para uma suinocultura, onde foi observada a preocupação de realizar tanto a destinação correta dos resíduos, quanto o seu reaproveitamento, quando possível, além dos cuidados com a segurança do trabalho. Foi realizado um estudo na Granja Bordignon, localizada no município de Campo Verde, Mato Grosso.

Palavras-chave:SGA, segurança do trabalho, resíduos de suinocultura.

Abstract: Waste is all substances considered undesirable generated in the production or consumption of goods. Through the development of an Environmental Management System (EMS), can be managed a productive system in order to properly allocate the waste produced, moreover, there is waste which can be reused for new activities. The correct management should consider the environmental and social issues in an integrated manner, thus, working security conditions are essential factor for proper operation. The solid waste produced in Bordignon Farm are separated and sent to appropriate disposal. Household waste is stored and sent to municipal collection, the recyclables are collected by municipal collectors Cooperative, the Health Services Waste are packed in for the companies specializing in disposal, the dead pigs and material from organic origin are sent to composting, the waste of pigs are sent to the wastewater treatment system, consisting of a Biodigestor followed by two lagoons. The gas generated in the digester is intended for a burner type Flaar and the final effluent resulting system this process is used for the organic fertilizer.This research aims to present an Environmental Management System - EMS realized for swine, where it observed the concern of performing both the proper disposal of waste, as its reuse, besides care safety. A study was conducted in Bordignon Farm, located in the municipality of Campo Verde, Mato Grosso.

Keywords:EMS, workplace safety, swine waste.

\section{INTRODUÇÃO}

Pode-se entender segurança do trabalho como associado de medidas que são tomadas com a finalidade de reduzir os acidentes de trabalho, doenças ocupacionais, bem como proteger a integridade e a capacidade laboral. A segurança do trabalho é estabelecida por normas e leis. No Brasil a legislação de segurança no trabalho baseiase na constituição federal, na consolidação das leis do trabalho, nas normas regulamentadoras e outras leis complementares, como portarias e decretos, e também as convenções internacionais da 
Citação:Brito J.;Zaque L.;Castro B.;Figueiredo K.;Guerra S.;Silveira J.Analyze of anEnvironmentSystem Management forSwine andObservation ofworkingconditions.E\&S - Engineering and Science, (2016).

Organização Internacional do Trabalho, aceitas pelo Brasil (PEIXOTO, 2011).

Conforme a Norma Regulamentadora 4 - Serviços Especializados em Engenharia de Segurança e em Medicina do Trabalho, as empresas privadas e públicas, os órgãos públicos da administração direta e indireta e dos poderes Legislativo e Judiciário, que possuam empregados regidos pela Consolidação das Leis do Trabalho - CLT, devem manter, obrigatoriamente, Serviços Especializados de Segurança e em Medicina do Trabalho, com finalidade de promover a saúde e proteger a integridade do trabalhador no local de trabalho.

De acordo com a Norma Regulamentadora 6 - Equipamento de Proteção Individual - EPI, considera-se Equipamento de Proteção Individual, todo dispositivo ou produto, de uso pessoal pelo trabalhador, indicado à proteção de riscos suscetíveis de ameaçar a segurança e saúde no trabalho. Ainda segundo a norma entende-se como equipamento conjugado de proteção individual, todo aquele composto por vários dispositivos, que o fabricante tem associado contra um ou mais riscos que pode ocorrer simultaneamente e que sejam suscetíveis de ameaçar a segurança e a saúde no trabalho.

Resíduos são todas as substâncias consideradas indesejadas geradas na produção ou consumo de bens, ou seja, ninguém quer produzir resíduos. Entretanto, a massa de resíduos sólidos produzida pela sociedade industrial é muito elevada devido à massa de produtos consumidos: todos os bens que consumimos ao final da sua vida útil serão resíduos; todo e qualquer processo de mineração, extração ou industrial produz resíduos (RIBEIRO \& MORELLI,2009).

Segundo a Norma Brasileira 10004 de 2004 - Resíduos Sólidos são: "Resíduos nos estados sólido e semissólido, que resultam de atividades de origem industrial, doméstica, hospitalar, comercial, agrícola, de serviços e de varrição. Ficam incluídos nesta definição os lodos provenientes de sistemas de tratamento de água, aqueles gerados em equipamentos e instalações de controle de poluição, bem como determinados líquidos cujas particularidades tornem inviável o seu lançamento na rede pública de esgotos ou corpos de água, ou exijam para isso soluções técnicas e economicamente inviáveis em face à melhor tecnologia disponível”.

Com o advento das revoluções Industriaise o crescimento desordenado e sem qualquer preocupação com o meio ambiente, os resíduospassaram a ser um problema de toda a humanidade. o desenvolvimento tecnológico, produzido para o conforto e o bem-estar humanos, gerado a partir dessas revoluções, ocasionou o aumento do material descartado, trazendo um aumento da quantidade de resíduos produzidos e não aproveitados, muitos deles ocasionando a contaminação do meio ambiente e do lençol freático, causando riscos à saúde humana, principalmente nas áreas urbanas. A maioria dos produtos é inutilizada e jogada fora com grande rapidez, devido ao fato que a humanidade passou a viver a era dos descartáveis (RIBEIRO, 2011).

De acordo com Cavalcanti (1990), pode-se dizer que a década de 70 foi a década da água, de 80 foi a década do ar e de 90a dos resíduos sólidos. Entretanto, não foi apenas no Brasil, nos EUA também começou a abordagem relativa a resíduos sólidos, porém somente no limiar da década de 80, quando foi estabelecido o Superfund, que era uma legislação específica que pretendia recuperar os grandes lixões de resíduos sólidos que existiam e ainda estãoespalhados nos EUA. Apesar de o Brasil estar em um momento histórico de progresso econômico, o alto consumo acarreta um aumento dos impactos ambientais em todas as fases supracitadas do sistema linear de produção, e tem como consequências a adição de geração de resíduos. Nesse cenário, os resíduos 
Citação:Brito J.;Zaque L.;Castro B.;Figueiredo K.;Guerra S.;Silveira J.Analyze of anEnvironmentSystem Management forSwine andObservation ofworkingconditions.E\&S - Engineering and Science, (2016).

industriais historicamente vêm sendo depositados de modo inapropriado no Brasil, na maioria das vezes, sem segregação (PAIXÃO et al., 2011).

A deposição de resíduos industriais, agrotóxicos, lixo e esgoto na natureza, são os meios predominantes da contaminação da água. Com essa contaminação também acontece a devastação das matas ciliares, provocando o assoreamento dos rios. As matas ciliares são as vegetações que cercam o curso dos rios, e sendo devastadas, deixam as margens dos mananciais sem proteção podendo rapidamente ser levadas pela água da chuva, causando a erosão (FERNANDES et al., 2007).

Em relação ao aspecto ambiental, a destinação imprópria de resíduos em lixões provoca degradação do meio ambiente, com a contaminação dos recursos naturais como o ar, solo, águas superficiais e subterrâneas. $\mathrm{O}$ tratamento e a destinação final dos resíduos são feitos através de respostas instantâneas, na maioria das vezes fundamentadas no simples descarte, prevalecendo os depósitos a céu aberto que contribuem para a deterioração ambiental (BROLLO e SILVA, 2001).

Conforme Silva et al.; (2012), os procedimentos de contaminação no solo acontecem vagarosamente e, frequentemente, sem consequências imediatas, entretanto, em longo prazo, podem ter efeitos sérios e, provavelmente irreversíveis. Os contaminantes podem resultar da degradação ou percolação das águas pluviais por resíduos sólidos (chorume ou percolado).

De acordo com a NBR ISO140012004, as normas de gestão ambiental têm por objetivo prover às organizações os elementos de um sistema de gestão ambiental (SGA) eficaz que possam ser integrados a outros requisitos de gestão, e auxiliá-las a alcançar seus objetivos ambientais e econômicos. A norma especifica os requisitos para que um sistema da gestão ambiental capacite uma organização a desenvolver e implementar política e objetivos que levem em consideração requisitos legais e informações sobre aspectos ambientais significativos.

O Sistema de Gestão Ambiental SGA é o modo pelo qual as instituições se mobilizam, externamente e internamente para o alcance do desempenho ambiental almejado (MARQUES et al. 2014).

Segundo Lazaretti et al., (2013), que fizeram um levantamento em 6 granjas, verificaram que são usados múltiplos modos de tratamento, como a utilização de composteiras, do ato de enterrar, de fossas sépticas, de lagoas e de biodigestores. E constataram que granjas participantes de projetos de integração são mais técnicas, e investem de forma mais apropriada na redução dos processos que acarretam prejuízos a natureza. Ainda conforme os autores, a utilização de biodigestores embora tenha seu custo de implantação, agrega receita à atividade pela venda de créditos de carbono. Uma dificuldade encontrada no trabalho foi em relação ao êxito dos contatos para a obtenção das informações e coleta de dados, ocorrendo ainda uma rejeição por parte dos produtores, mesmo com a garantia da confidencialidade dos dados.

Conforme Oliveira e Nunes (2005), os dejetos devem ser usados totalmente na agricultura como fertilizante orgânico, buscando-se aplicar os nutrientes nos dejetos impedindo o desperdício d'água nos sistemas de produção. Os dejetos líquidos excedentes nas propriedades devem ser tratados corretamente, utilizando-se o resíduo final para a limpeza dos dejetos impedindo o seu lançamento nos cursos d'água. Ainda segundo os autores, a utilização de compostagem para o tratamento de dejetos de suínos vem sendo uma opção usada essencialmente em regiões de produção intensiva de suínos, gerando um composto orgânico estabilizado que 
Citação:Brito J.;Zaque L.;Castro B.;Figueiredo K.;Guerra S.;Silveira J.Analyze of anEnvironmentSystem Management forSwine andObservation ofworkingconditions.E\&S - Engineering and Science, (2016).

pode ser empregado como fertilizante orgânico.

Este trabalho tem como objetivo apresentar um sistema de gestão ambiental para uma suinocultura visando à destinação correta dos resíduos da região de Campo Verde e observar o ambiente físico e social, além da segurança dos trabalhadores.

\section{MATERIAL E MÉTODOS}

O trabalho foi realizado na Granja Bordignon localizada no município de Campo Verde - MT no Km 06 Zona Rural, MT 344, com a latitude $15^{\circ} 33^{\prime} 50^{\prime \prime}$ e longitude $55^{\circ} 08^{\prime} 50^{\prime \prime}$, alojada 500 matrizes em regime de confinamento intensivo, em ciclo completo (produção de leitões e engorda), a propriedade da granja é de aproximadamente 11 hectares, composta por nove (09) funcionários e três (03) proprietários.

Utilizou-se registros fotográficose visitas in loco da área de estudo nos anos de
2014 e 2015, foram observadas, condições ambientais e de segurança do trabalho e seu sistema de gestão.

Figura 1 - Localização Granja Bordignon

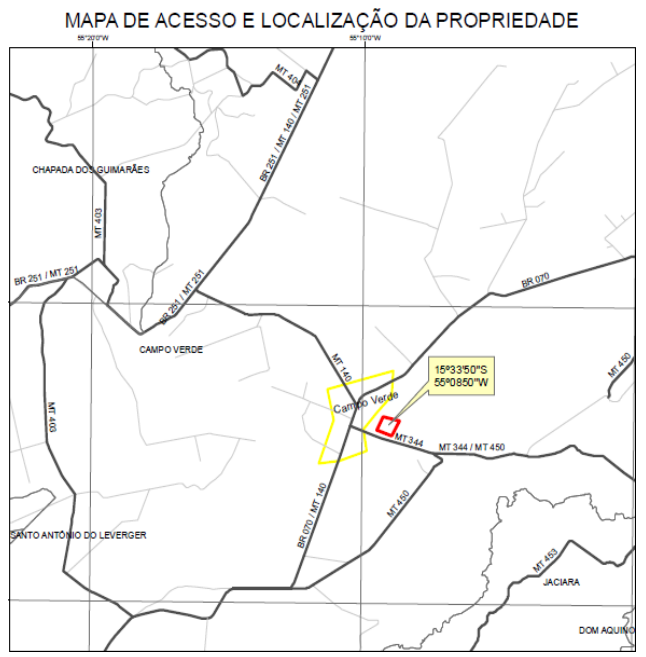

Fonte: Brito J. (2015)

\section{RESULTADOS E DISCUSSÕES}

Resultados apresentadas na fertirrigação:

NoQuadro 1pesquisas realizadas na área de fertirrigação da Granja Bordignom, MT (2013/2015):

Quadro 1 -Apresentação de gestão de resíduose $\quad$ EPIs utilizados

\begin{tabular}{|l|l|l|l|}
\hline RESÍDUOS & $\begin{array}{l}\text { METODOLOGIA / } \\
\text { TRATAMENTO }\end{array}$ & DESTINAÇÃO & EPI UTILIZADO \\
\hline $\begin{array}{l}\text { Efluente líquido } \\
\text { da produção de } \\
\text { suínos }\end{array}$ & $\begin{array}{l}\text { Estação de tratamento } \\
\text { de esgoto } \\
\text { (gradeamento, } \\
\text { biodigestor, lagoas) } \\
\text { fertirrigação }\end{array}$ & $\begin{array}{l}\text { Sistema de } \\
\text { tratamento e } \\
\text { disposiçãono solo } \\
\text { agrícola }\end{array}$ & $\begin{array}{l}\text { Mascara, luva, óculos, bota de borracha, } \\
\text { chapéu, protetor solar,macacao de } \\
\text { manga comprida }\end{array}$ \\
\hline Recicláveis & Triagem e comércio & $\begin{array}{l}\text { Cooperativa de } \\
\text { catadores }\end{array}$ & $\begin{array}{l}\text { luva, óculos, bota de borracha, chapeu, } \\
\text { protetorsolar,macacao de manga } \\
\text { comprida }\end{array}$ \\
\hline $\begin{array}{l}\text { Resíduos de } \\
\text { serviços de } \\
\text { saúde (RSS) }\end{array}$ & $\begin{array}{l}\text { Incineração, } \\
\text { autoclavagem }\end{array}$ & $\begin{array}{l}\text { Empresas } \\
\text { comlicenças de } \\
\text { coleta }\end{array}$ & Luva,ocúlos,aventalimpermeável,chapéu \\
\hline $\begin{array}{l}\text { Suínosmortos } \\
\text { compostagem }\end{array}$ & $\begin{array}{l}\text { Aduboorgânico / } \\
\text { fertilizante }\end{array}$ & $\begin{array}{l}\text { Aterro sanitário } \\
\text { municipal }\end{array}$ & $\begin{array}{l}\text { Luva,bota de proteção,calça e blusa de } \\
\text { manga comprida }\end{array}$ \\
\hline $\begin{array}{l}\text { Resíduos } \\
\text { domésticos }\end{array}$ & Coleta municipal & \\
\hline
\end{tabular}


Citação:Brito J.;Zaque L.;Castro B.;Figueiredo K.;Guerra S.;Silveira J.Analyze of anEnvironmentSystem Management forSwine andObservation ofworkingconditions.E\&S - Engineering and Science, (2016).

$\mathrm{Na} \quad$ Figura 2 é apresentada acomposteira de suínos mortos, onde após o período de 120 dias o material está pronto para ser usado na agricultura.

Figura 2 - Adubaçãocomdejetos de suínos.

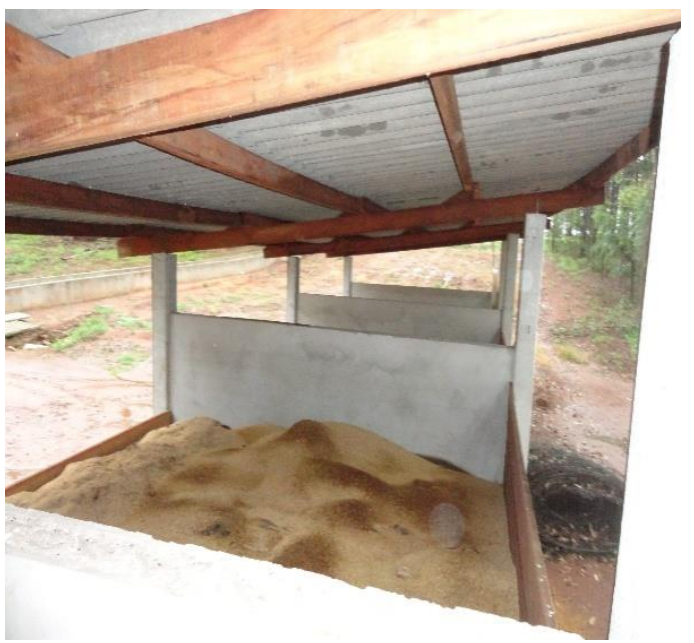

Fonte: Brito J. (2015)

Resultados apresentados em segurança do trabalho $e$ alguns aspectos ambientais dentro da granja

Foi verificado uso de Equipamento de Proteção Individual - EPI, pertinente ao trabalho desenvolvido por parte da equipe de trabalho. Segundo os administradores da Granja, há resistência por parte dos colaboradores quanto ao uso de alguns EPI, (colete, óculos e macacão de borracha) debido ao calor e o desconforto.

$\begin{array}{cccr}\text { De } & \text { acordocom } & \text { a } & \text { Norma } \\ \text { Regulamentadora } 4 & - & \text { Serviços }\end{array}$

Especializados em Engenharia de Segurança e em Medicina do Trabalho, as atividades econômicas de criação de suínos, cultivo de cereais e produção de florestas plantadas apresentam grau de risco 3.
A granja nãopossui CIPA por ser composta de 12 colaboradores sendo 09 funcionários e 03 proprietários, sendoassim, conforme a Norma Regulamentadora 5 Comissão Interna de Prevenção de Acidentes - CIPA, para seestabelecer a CIPA é necessário ter pelo menos 20 funcionários.

Caso no decorrer dos anos a Granja Bordignonpassar a ter mais de 19 funcionários, o treinamento da CIPA deverá ter o estudo do ambiente, condições de trabalho e riscos originados do processoprodutivo.

Emrelaçãoao ambiente pode ser dividido em ambiente físico e ambiente social. O ambiente físico apresentaemgeral boas condições de limpeza e iluminação noslocais de trabalho, a Granja Bordignon é bem constituida quanto à organização do seu espaço físico. O ambiente social é saudável entre os funcionários e os proprietários, já que todos expressam opiniões a respeito do trabalho.

Em relação às condições de trabalho há varias sinalizações e placas entre os setores de trabalho conforme apresentados nas Figuras 3 a e b. A pesar de ter EPI disponível, alguns funcionários não utilizam em determinados setores, só utilizam quando é extremamente necessário. De acordocom a Norma Regulamentadora 6 - Equipamentos de Proteção Individual, a empresa é obrigada a fornecer aos empregados, gratuitamente, EPI adequado ao risco, em perfeito estado de conservação e funcionamento, e a Granja Bordignon fornece-os. 
Citação:Brito J.;Zaque L.;Castro B.;Figueiredo K.;Guerra S.;Silveira J.Analyze of anEnvironmentSystem Management forSwine andObservation ofworkingconditions.E\&S - Engineering and Science, (2016).

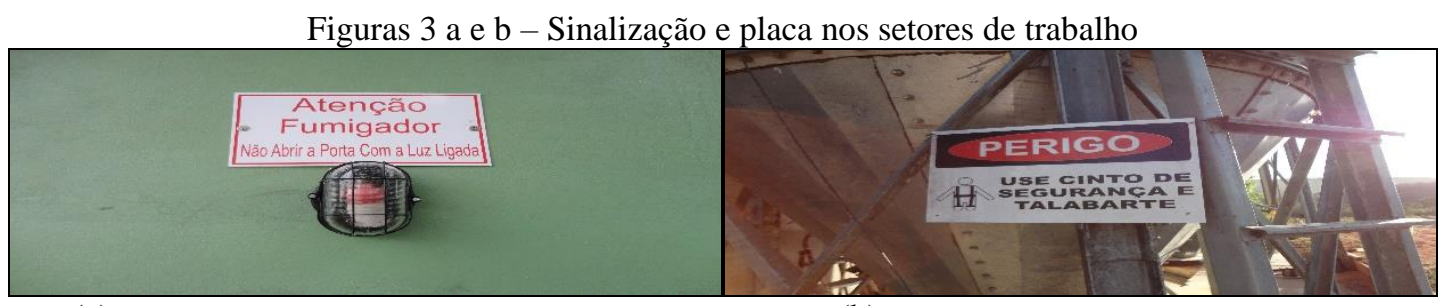

(a)

(b)

Fonte: Brito J. (2015)

O proceso produtivo pode causar danos tanto para o trabalhador quanto para o meio ambiente, poisalguns dos residuos

gerados são tóxicos podendo contaminar o solo atingindo o lençol freático como mostrados nasFiguras $4 \mathrm{a} \mathrm{b}$.

Figuras 4 a e b - Chegada dos dejetos a lagoa, ondetem-se cerca de ummilhão de litros. A lagoa é usada para fertirrigação que através de uma bomba de auto pressão, pulveriza a plantação com uma tubulação comum "canhão" na ponta

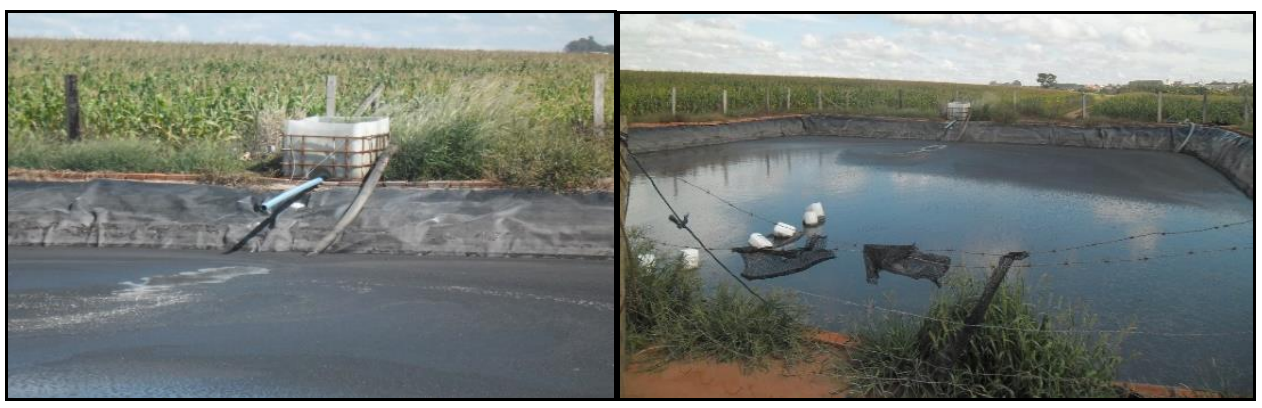

(a)

(b)

Fonte: Brito J. (2015)

Conforme a Norma Regulamentadora 7 - Programa de Controle Médico de Saúde Ocupacional - PCMSO, para empresas que tenham mais de 10 e com até 20 funcionários poderão estar desobrigadas de indicar médico do trabalho coordenador em decorrência de negociação coletiva, assistida por profissional do órgão regional competente em segurança e saúde no trabalho, então ficaráa o critério da empresa indicar médico do trabalho.

$\mathrm{Na}$ Granja Bordignon, os funcionarios trabalham com animai sem estado deteriorado e segundo a Norma Regulamentadora $15-$ Atividade e Operações Insalubres, resíduos de animais deteriorados são consideradas de Insalubridade de grau médio, como mostram as Figuras 5 a e b.

Figuras 5 a e b - Depósito final dos resíduos, antes da retirada da prefeitura e composteira de suínosmortos.

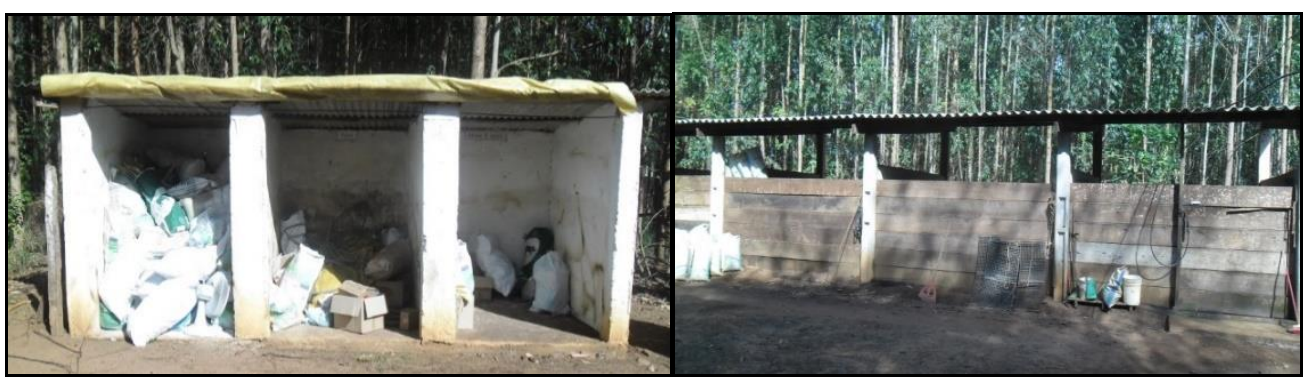

Fonte: Brito J. (2015)

(a)

(b) 
Citação:Brito J.;Zaque L.;Castro B.;Figueiredo K.;Guerra S.;Silveira J.Analyze of anEnvironmentSystem Management forSwine andObservation ofworkingconditions.E\&S - Engineering and Science, (2016).

De acordo com a Norma Regulamentadora 16 - Atividades e Operações Perigosas, a Granja Bordignon apresenta atividades ou operações perigosas, pois apresenta biodigestor que pode causar explosão.

Figura 6 - Biodigestor

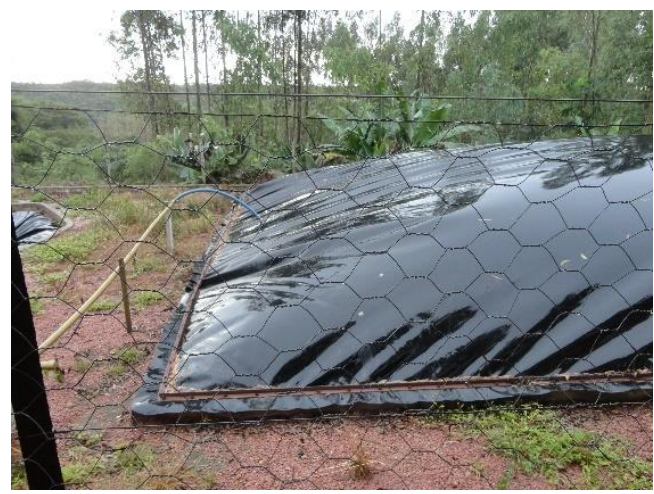

Fonte: Brito J. (2015)

Em relação ao desempenho de suas funções, os funcionários da Granja Bordignon trabaran segundo as Mrs. e apresentam condições adequadas quanto àscondições ergonômicas, pois de acordocom a Norma Regulamentadora 17 Ergonomia, os funcionarios recebem treinamento em relação aos métodos de trabalho evitando assim acidentes inesperados. Para transporte manual oufeito por equipamento são realizados por funcionários que têm condições físicas e psicológicas para fazê-las.

\section{CONCLUSÃO}

O sistema de gestão ambiental para a suinocultura da Granja Bordinon apresentado é adequado, pois utiliza os resíduos para adubar as culturas agrícolas. $\mathrm{O}$ ambiente social é saudável, pois há respeito entre os superiores e subordinados, onde todos procuram ajudar o próximo. $\mathrm{O}$ ambiente físico é um local limpo, arejado e sinalizado, favorecendo condições adequadas para o desenvolvimento do trabalho. Apesar de alguns trabalhadores terem resistência quanto ao uso completo de EPI, devido ao calor e desconforto, a grande maioria dos trabalhadores utiliza os EPIs ocorrendo menos acidentes de trabalho.

\section{RECOMENDAÇÕES}

É recomendado que a Granja sempre possua EPI reservas, caso ocorra algum problema com os EPI que estão sendo utilizados.É importante, também, que incentivem mais os trabalhadores a utilizarem os EPI. Recomendamos que o (supervisor, gerente de pátio) fique atento com a utilização dos Equipamentos de Proteção Individual (EPI), para saber se todos estão usando, conferir a validade, se estão higienizando e usando de maneira correta, bem como mostrar a importância de sua utilização.

\section{AGRADECIMENTOS}

Agradecemos aos proprietários e gerentes da Granja Bordignon por permitir a visita para a realização deste trabalho e autorizar divulgação dos principais dados levantados. A empresa CSA Consultoria e Serviços Ambientais. A Professora Margarida Machetto pelo seu incentivo e colaboração.

\section{REFERÊNCIAS BIBLIOGRÁFICAS}

ABNT, 2004.NBR 10004. Resíduos sólidos Classificação.

ABNT, 2004.NBR 14001. Sistema de gestão ambiental - Requisitos com orientações para uso.
BROLLO, M. J.; SILVA, M. M. Política e Gestão Ambiental em Resíduos Sólidos. Revisão e análise sobre a atual situação no Brasil. $21^{\circ}$ Congresso Brasileiro de Engenharia Sanitária e Ambiental. ABES - Trabalhos Técnicos. 2001. 
Citação:Brito J.;Zaque L.;Castro B.;Figueiredo K.;Guerra S.;Silveira J.Analyze of anEnvironmentSystem Management forSwine andObservation ofworkingconditions.E\&S - Engineering and Science, (2016).

$<$ http://www.researchgate.net/publication/228885 347_POLTICA_E_GESTO_AMBIENTAL_EM_ RESDUOS_SLIDOS._REVISO_E_ANLISE_SO BRE_A_ATUAL_SITUAO_NO_BRASIL>.

Acesso: Jul. 2015.

CAVALCANTI, J. E. A década de 90 é dos resíduos sólidos. Revista Saneamento Ambiental - $\mathrm{n}^{\mathrm{o}}$ 54, p. 16-24, nov./dez. 1998. Disponível em: < http://www.riosvivos.org.br/Noticia/A+decada+d e+90+e+dos+residuos+solidos/3223>. Acesso: Jul. 2015.

FERNANDES, D. R. M.; et al. Viabilidade econômica do uso água da chuva: um estudo de caso da implantação de cisterna na UFRN/RN. XXVII Encontro Nacional de Produção. A energia que move a produção: um diálogo sobre integração, projeto e sustentabilidade. Foz do Iguaçu, PR, Brasil. $2007 . \quad$ Disponível em: <http://www.abepro.org.br/biblioteca/ENEGEP2 007_TR650479_0552.pdf>. Acesso: Jul. 2015.

LAZARETTI, R. M. J.; SANTOS, J. M. G dos; ANDREAZZI, M. A. Destinação dos resíduos da suinocultura em granjas das regiões Sudoeste, Norte e Noroeste do estado do Paraná. VII Encontro Internacional de Produção Científica. UNICESUMAR Centro Universitário Cesuram. Maringá Paraná. 2013. Disponível em: <http://www.cesumar.br/prppge/pesquisa/epc c2013/oit_mostra/Rhubia_Maria_Jorge_Laza retti.pdf>. Acesso: 22 Dez 2015.

MARQUES M.; et al. A Percepção Ambiental pelos Alunos da UFMT- Campus Cuiabá como Instrumento de Planejamento de Sistemas de Gestão Ambiental nas Empresas. E\&S Engineeringand Science, (2014), 2:1. Disponível em: $<$ http://periodicoscientificos.ufmt.br/index.php/e ng/article/view/2070>. Acesso: jul. 2015.

MTE. Ministério do Trabalho e Emprego. Portaria SIT No 2018 de 23 de DEZ 2014. NR 4. Serviços Especializados em Engenharia de Segurança e em Medicina do Trabalho.

MTE. Ministério do Trabalho e Emprego. Portaria SIT No 247 de 12 de JUL 2011. NR 5. Comissão Interna de Prevenção de Acidentes.
MTE. Ministério do Trabalho e Emprego. Portaria SIT No 292 de 08 de DEZ 2011. NR 6. Equipamentos de Proteção Individual - EPI.

MTE. Ministério do Trabalho e Emprego. Portaria MTE N ${ }^{\circ} 1892$ de 09 de DEZ 2013. NR 7. Programa de Controle Médico de Saúde Ocupacional.

MTE. Ministério do Trabalho e Emprego. Portaria SIT No 291 de 08 de DEZ 2011. NR 15. Atividades e Operações insalubres.

MTE. Ministério do Trabalho e Emprego. Portaria MTE N ${ }^{\circ} 05$ de 07 de JAN 2015. NR 16. Atividades e Operações perigosas.

MTE. Ministério do Trabalho e Emprego. Portaria SIT N 13 de 21 de JUN 2007. NR 17. Ergonomia.

OLIVEIRA, P. A. V., NUNES, M. L. A. Sustentabilidade ambiental da suinocultura. Workshop sobre Tecnologias para a Remoção de Nutrientes de Dejetos de Origem Animal. Embrapa Suínos e Aves - Santa Catarina. 2005. Disponível em: <http://www.cnpsa.embrapa.br/sgc/sgc_publicac oes/anais0205_oliveira.pdf>.

PAIXÃO, J. F. da. ROMA, J. C.; MOURA, A. M. M. Resíduos Sólidos Industriais. Caderno de diagnóstico. 2011. 82 p. Disponível em: < http://www.cidadessustentaveis.org.br/sites/defau lt/files/arquivos/05_residuos_solidos_industriais_ 0.pdf>. Acesso: jul. 2015.

PEIXOTO, N. H. Curso técnico em automação industrial: segurança do trabalho. 3. Ed. Santa Maria: Universidade Federal de Santa Maria: Colégio Técnico Industrial de Santa Maria, 2011. 128p.

RIBEIRO, D. V. Apolítica nacional de resíduos sólidos e o uso indiscriminado de resíduos sólidos pela sociedade brasileira. Universidade Federal de São Carlos. 2011. 12 p. Disponível em:

<http://www.sustenta2013.com/arquivos/ARTIG O_DANIEL_VERAS.pdf >. Acesso: jul. 2015.

RIBEIRO, D.V.; MORELLI, M.R. Resíduos Sólidos: problema ou oportunidade? $1^{\mathrm{a}}$ Edição. Rio de Janeiro: Editora Inter ciência, 2009. 158 p. 
Citação:Brito J.;Zaque L.;Castro B.;Figueiredo K.;Guerra S.;Silveira J.Analyze of anEnvironmentSystem Management forSwine andObservation ofworkingconditions.E\&S - Engineering and Science, (2016).

SILVA, C da.; et al. Diagnostico da contaminação do solo e aplicação do índice de qualidade de aterros de resíduos da CESTESB na área de disposição de resíduos sólidos urbanos de Peabirú-PR. Revista Engenharia Ambiental. Espírito Santo do Pinal. v.9, n.2, p. 252-270, abr/jun. 2012. Disponível em: <http://webresol.org/textos/eapt-2011-737.pdf>. Acesso: Jul. 2015. 